\title{
Flora da Bahia: Typhaceae
}

\section{Maria Luiza Silveira de Carvalho ${ }^{*}$, Carla Teixeira de Lima ${ }^{2, a}$, Reyjane Patricia de Oliveira ${ }^{1,2, b} \&$ Ana Maria Giulietti ${ }^{2,3, c}$}

${ }^{1}$ Programa de Pós-Graduação em Genética e Biodiversidade, Universidade Federal da Bahia, Bahia, Brasil.

${ }^{2}$ Programa de Pós-Graduação em Botânica, Universidade Estadual de Feira de Santana, Bahia, Brasil.

${ }^{3}$ Royal Botanic Gardens, Kew, Reino Unido.

\begin{abstract}
Resumo - É apresentado o levantamento florístico de Typhaceae da Bahia, Brasil, como contribuição ao conhecimento da flora do Estado. São reconhecidas duas espécies de Typha: T. domingensis e T. latifolia. É apresentada uma chave para diferenciar as espécies, além de descrições e comentários gerais para os táxons, ilustrações e mapa de distribuição das espécies na Bahia.

Palavras-chave adicionais: Brasil, florística, plantas aquáticas, taxonomia, Typha.
\end{abstract}

Abstract (Flora of Bahia: Typhaceae) - We present a floristic survey of Typhaceae from Bahia State, Brazil, as a contribution to the knowledge of the Flora of the State. Two species are recognized in the genus Typha: T. domingensis and T. latifolia. We present a key to distinguish the species, descriptions and general comments for the taxa, illustrations and distribution map of species in Bahia.

Additional key words: aquatic plants, Brazil, floristics, taxonomy, Typha.

\section{TYPHACEAE}

Ervas perenes aquáticas ou de áreas brejosas e alagadiças; caule subterrâneo do tipo rizoma horizontal, glabro. Folhas sésseis, bainha aberta, limbo linear, nervação paralela; aerênquima presente. Flores unissexuadas, numerosas, reunidas em inflorescência ereta, terminal, emergente, em forma de espiga ou globosa, bráctea da inflorescência foliácea, geralmente decídua. Flores estaminadas numerosas, decíduas, ráquis persistente, geralmente separadas e em posição apical em relação às flores pistiladas, sésseis ou estipitadas; anteras basifixas. Flores pistiladas numerosas, persistentes até o fruto, sésseis ou estipitadas, hipóginas; ovário 3-carpelar, sincárpico, unilocular, uniovulado, branco, verde ou castanho, ginóforo presente; estilete 1; estigma 1. Fruto drupáceo ou aquênio, fusiforme ou elipsoide. Sementes com endosperma farináceo, amiláceo ou oleífero; embriões cilíndricos ou lineares.

Família cosmopolita, distribuída na região tropical até a temperada. Atualmente, são reconhecidos dois gêneros para a família: Typha L., com 8 a 13 espécies, e Sparganium L., com cerca de 14 espécies, encontradas principalmente no Hemisfério Norte (Dahlgren et al. 1985; Kubitzki 1998). Estudos filogenéticos baseados em dados morfológicos e moleculares (Duval et al. 1993; Chase et al. 1995; Stevenson \& Loconte 1995; Stevenson et al. 2000; Michelangeli et al. 2003; APG III 2009; Kim \& Choi 2011) sustentam Sparganium como grupo irmão de Typha. No Brasil, apenas Typha é nativo, sendo Sparganium cultivado (Reitz 1984; Bove 2010).

\footnotetext{
*Autora para correspondência: silveiradecarvalho@yahoo.com.br;

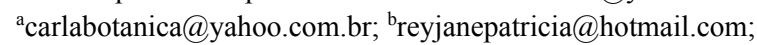
canagiulietti@hotmail.com

Editor Responsável: Pedro Fiaschi

Submetido: 16 maio 2013; aceito: 23 jul. 2014

Publicação eletrônica: 15 ago. 2014; versão final: 19 ago 2014
}

\section{Typha L.}

Ervas paludosas; rizoma rico em amido; catafilos caducos; caule aéreo cilíndrico, maciço. Folhas lineares, dísticas, retorcidas, plano-convexas, coriáceas ou esponjosas. Flores aclamídeas, em espiga terminal cilíndrica, contínua ou interrompida por parte estéril da ráquis. Flores pistiladas e estaminadas recobertas por tricomas resultantes da redução do perianto. Flores estaminadas em fileiras simples; estames (1-)3(-9); anteras 4-esporangiadas, oblongas ou romboides, deiscentes longitudinalmente. Flores pistiladas com ou sem brácteas; ginóforo com 10-50 tricomas, geralmente filiformes; ovário oblongo ou fusiforme, continuado em estilete simples; estigma alongado, linear a oblanceolado ou romboide; óvulo anátropo, pêndulo, bitegumentado. Fruto aquênio. Sementes amarelas; embrião ortótropo.

Typha tem distribuição cosmopolita, com ocorrência do Círculo Polar Ártico até latitudes acima de $30^{\circ}$ no Hemisfério Sul (Dahlgren et al. 1985; Kubitzki 1998; Stevens 2014). Para a Bahia, é citada a ocorrência apenas de T. domingensis (Bove 2010). No entanto, durante o desenvolvimento deste trabalho foram identificadas as duas espécies citadas para o Brasil, $T$. domingensis e T. latifolia, encontradas em áreas inundadas ou lagoas temporárias, ocorrendo inclusive juntas.

\section{Chave para as espécies}

1. Flores pistiladas com poucos tricomas (ca. 15) no ginóforo, estigma romboide; flores estaminadas com anteras oblongas ................. 1. T. domingensis

1'. Flores pistiladas com muitos tricomas (32-53) no ginóforo, estigma linear a oblanceolado; flores estaminadas com anteras romboides 
1. Typha domingensis Pers., Syn. P1. 2: 532. 1807. Figuras 1 e 2A-D.

Erva aquática e/ou paludosa, emergente, ereta, 1,1$4 \mathrm{~m}$ alt. Folhas sésseis, dísticas, coriáceas; lâmina linear, 34-120 × 0,5-1,5 cm, concolor ou ligeiramente glauca na face abaxial, ápice agudo, margem inteira, bainha aberta, glabra. Flores reunidas em espiga; a porção com flores estaminadas $19-35 \times 0,8-1,5 \mathrm{~cm}$; a porção com flores pistiladas $9-29 \times 1-2,5 \mathrm{~cm}$. Flores estaminadas com (1)2(-9) estames; anteras oblongas. Flores pistiladas com sépalas filiformes, $30-55 \mathrm{~mm}$ compr.; ovário 1-1,5 mm compr.; ginóforo $1-4,5 \mathrm{~mm}$ compr., com ca. 15 tricomas; estilete 1-3 mm compr.; estigma 1-2 mm compr., romboide. Fruto 1-15 mm compr.

Espécie com ampla distribuição nas Américas, ocorrendo no sul dos Estados Unidos, México, Antilhas, América Central, e em toda a América do Sul, até a Patagônia (Dahlgren et al. 1985; Kubitzki 1998). No Brasil, pode ser encontrada nas Regiões Norte, Nordeste, Centro-Oeste, Sudeste e Sul (Reitz 1984; Bove 2010). D6, D7, E6, E9, F8, G8: floresta atlântica, restinga, caatinga, cerrado e campo rupestre. Floresce durante o ano todo.

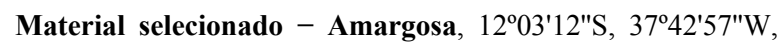
19 out. 2007 (fl.), J.L. Paixão 1379 (HUEFS); Candeias, BR-324, 12³8'45"S, 38²8'32"W, 2 nov. 2010 (fl.), E. Melo et al. 5290 (HUEFS); Capim Grosso, 11 ago. 2004 (fl.), A. Amaral-Santos 2433 (HUEFS); Feira de Santana, 17 maio 2007 (fl.), M.V. Moraes 807 (HUEFS); Ilhéus, nov. 1985 (fl.), R.M. Matos \& N.G. Jesus 139 (HRB); Jacobina, 12 out. 2007 (fl.), J.L. Ferreira 114 (HUEFS); Morro do Chapéu, rio Tareco, próximo ao balneário Oásis do Tareco, 1124'24"S, 41²2'19"W, 14 ago. 2009 (fl.), C.T. Lima et al. 192 (HUEFS); Piritiba, 31 maio 1980 (fl.), L.R. Noblick 1891 a (HUNEB); Salvador, nov. 2005 (fl.), L.P. Queiroz et al. 1278 (HRB); Santa Luz, fazenda Fontinha, tanque do Governo, $11^{\circ} 17^{\prime} 21^{\prime \prime S}, 39^{\circ} 44^{\prime} 54^{\prime \prime W}, 4$ set. 2009 (fl.) C.T. Lima et al. 223 (HUEFS); Santo Antônio de Jesus, rodovia entre Santo Antônio de Jesus e Gandu, 2 ago. 2009 (fl.), C.T. Lima et al. 190 (HUEFS).

Typha domingensis difere de T. latifolia por possuir flores mais delicadas, com menor quantidade de tricomas no ginóforo, estigmas romboides e anteras oblongas.

\section{Typha latifolia L., Sp. Pl. 2: 971. 1753.}

Figuras 1 e 2E-H.

Erva aquática, emergente, ereta ou subereta, 1-3 m alt. Folhas sésseis, dísticas, coriáceas; lâmina linear, 45-120 × 0,6-2 cm, concolores, ápice agudo, margem inteira, bainha aberta, glabra. Flores reunidas em espigas; a porção com flores estaminadas 10-28 $\times 0,7$ $1,5 \mathrm{~cm}$; a porção com flores pistiladas $9-29 \times 1-2,5$ $\mathrm{cm}$. Flores estaminadas com 2 ou 3(-7) estames; anteras romboides. Flores pistiladas com sépalas filiformes, $30-55 \mathrm{~mm}$ compr.; ovário $0,7-1,5 \mathrm{~mm}$ compr.; ginóforo 2,5-6 $\mathrm{mm}$ compr., com 32-53

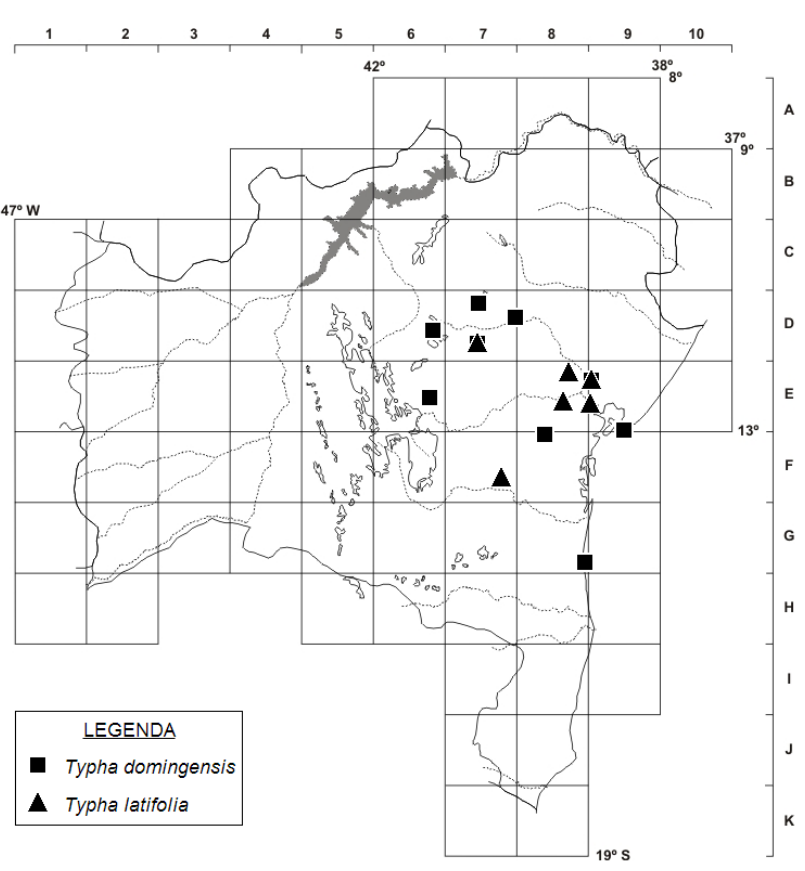

Figura 1. Mapa de distribuição de Typha domingensis e T. latifolia no estado da Bahia, Brasil.

tricomas; estilete $2-5,5 \mathrm{~mm}$ alt.; estigma $0,5-1 \mathrm{~mm}$ compr., linear a oblanceolado. Fruto $1-15 \mathrm{~mm}$ compr.

Distribuída dos trópicos até a região temperada, exceto na Ásia Meridional, Austrália e Polinésia (Dahlgren et al. 1985; Kubitzki 1998). No Brasil, é encontrada nas Regiões Norte, Nordeste e Sudeste (Reitz 1984; Bove 2010). D7, E8, E9, F7: floresta atlântica, campo rupestre e restinga. Floresce durante $\mathrm{o}$ ano inteiro.

Material selecionado - Andaraí, Pantanal de Marimbus, 12 $45^{\prime} 47^{\prime \prime S}, 41^{\circ} 18^{\prime} 33^{\prime \prime W, ~} 22$ ago. 2009 (bot.), C.T. Lima et al. 211 (HUEFS); Anguera, 28 jan. 1997 (fl.), E. Melo 2011 (HUEFS); Cachoeira, nov. 1980 (fl.), L. Scardino 862 (HUNEB); Candeias, 21 out. 2004 (fl.), K.R.B. Leite 470 (HUEFS); Feira de Santana, 7 set. 1983 (fl.), L.R. Noblick 2730 (HUEFS); Lafaiete Coutinho, maio 2002 (fl.), L.C. Senra 27 (HUNEB); Piritiba, 31 maio 1980 (fl.), L.R. Noblick $1891 b$ (HUNEB).

Typha latifolia apresenta flores com mais tricomas no ginóforo do que $T$. domingensis, os estigmas são lineares a oblanceolados e os estames são romboides. De acordo com observações de campo, T. latifolia possui antese noturna (C.T. Lima, obs. pess.).

\section{AGRADECIMENTOS}

Os autores agradecem ao CNPq e à CAPES pelas bolsas de doutorado e pós-doutorado concedidas à MLSC; à CAPES pela bolsa de doutorado à CTL; ao $\mathrm{CNPq}$ pelas bolsas de produtividade em pesquisa para RPO e AMG; ao MCT e à Fapesb pelo apoio financeiro aos projetos PPBio do Semi-árido e Flora da Bahia, respectivamente. 

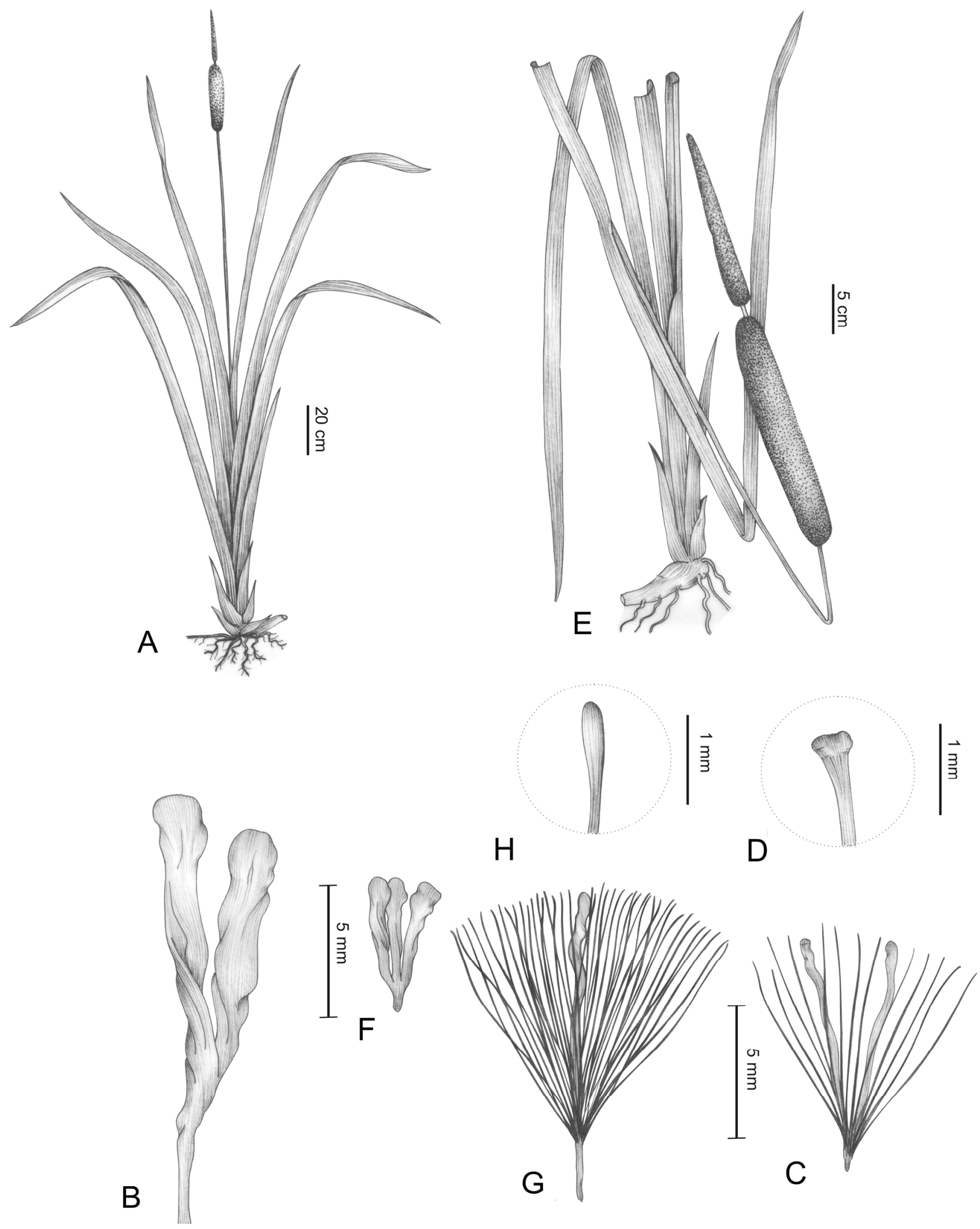

Figura 2. A-D. Typha domingensis: A- hábito; B- estames; C- duas flores pistiladas; D- detalhe do estigma. E-H. Typha latifolia: E- hábito com detalhe da inflorescência; F- estames; G- flor pistilada; H- detalhe do estigma (A-D- Lima 192; E e F- Lima 211; G e H- Lima 1686).

\section{REFERÊNCIAS}

APG III 2009. An update of the Angiosperm Phylogeny Group classification for the orders and families of flowering plants: APG III. Botanical Journal of the Linnean Society 161: 105-121.
Bove, C.P. 2010. Typhaceae. In: R.C. Forzza, J.F.A. Baumgratz, C.E.M. Bicudo, A.A. Carvalho Jr, A. Costa, D.P. Costa, M. Hopkins, P.M. Leitman, L.G. Lohmann, L.C. Maia, G. Martinelli, M. Menezes, M.P. Morim, M.A.N. Coelho, A.L. Peixoto, J.R. Pirani, J. Prado, L.P. Queiroz, V.C. Souza, J.R. 
Stehmann, L.S. Sylvestre, B.M.T. Walter \& D. Zappi (eds), Catálogo de Plantas e Fungos do Brasil. Vol. 1. Instituto de Pesquisas Jardim Botânico do Rio de Janeiro, Rio de Janeiro, p. 1332.

Chase, M.W.; Duvall, M.R.; Hills, H.G.; Conran, J.G.; Cox, A.V.; Eguiarte, L.E.; Hartwell, J.; Fay, M.F.; Caddick, L.R.; Cameron, K.M. \& Hoot, S. 1995. Molecular phylogenetics of Lilianae. In: P.J. Rudall, P.J. Cribb, D.F. Cutler \& C.J. Humphries (eds), Monocotyledons: systematics and evolution. Royal Botanic Gardens, Kew, p. 109-137.

Dahlgren, M.R.T.; Clifford, H.T \& Yeo, F. 1985. Typhaceae. In: M.R.T. Dahlgren, H.T Clifford \& F. Yeo (eds), The Families of the Monocotyledons. Springer Verlag, Berlin, p. 348-349.

Duval, M.R.; Clegg, M.T.; Chase, M.W.; Lark, W.D.; Kress, W.J.; Hills, H.D.; Eguiarte, L.E.; Smith, J.F.; Gaut, B.S.; Zimmer, E.A. \& Learn Jr., G.H. 1993. Phylogenetic hypotheses for the monocotyledons constructed from $r b c L$ sequences. Annals of the Missouri Botanical Garden 80: 607-619.

Kim, K. \& Choi, H.K. 2011. Molecular systematics and character evolution of Typha (Typhaceae) inferred from nuclear and plastid DNA sequence data. Taxon 60: 1417-1428.

Kubitzki, K. 1998. Typhaceae. In: K. Kubitzki (ed.), The Families and Genera of Vascular Plants. IV. Flowering plants: monocotyledons. Alismatanae and Commelinanae (except Gramineae). Springer Verlag, Berlin, p. 457-460.

Michelangeli, F.A.; Davis, J.I. \& Stevenson, D.W. 2003. Phylogenetic relationships among Poaceae and related families as inferred from morphology, inversions in the plastid genome, and sequence data from the mitochondrial and plastid genomes. American Journal of Botany 90: 93-106.

Reitz, R. 1984. Tifáceas. In: R. Reitz (ed.), Flora Ilustrada Catarinense. Vol.1. Herbário Barbosa Rodrigues, Itajaí.

Stevens, P. 2014. Angiosperm Phylogeny Website. Missouri Botanical Gardens. Disponível em <http://www.mobot.org/ mobot/research/apweb>; acesso em 15 jun. 2014.

Stevenson, D.W. \& Loconte, H. 1995. Cladistic analysis of monocot families. In: P.J. Rudall, P.J. Cribb, D.F. Cutler \& C.J. Humphries (eds), Monocotyledons: systematics and evolution. Royal Botanic Gardens, Kew, p. 543-578.

Stevenson, D.W.; Davis, J.I.; Freudenstein, J.V.; Hardy, C.R.; Simmons, M.P. \& Specht, C.D. 2000. A phylogenetic analysis of the monocotyledons based on morphological and molecular character sets, with comments on the placement of Acorus and Hydatellaceae. In: K.L. Wilson \& D.A. Morrison (eds), Monocots: systematics and evolution. CSIRO, Melbourne, p. $17-24$.

\section{LISTA DE EXSICATAS}

Adorno, J.M.S. 1 (1); Amaral-Santos, A. 2433 (1); Andrade, M.J.G. 618 (1); Dambrós, L.A. 187 (1); Ferreira, J.L. 114 (1); França, F. 1889 (2), 3111 (1); Giordano, L.C. 476 (1); Giulietti, A.M. 2041 (2); Leite, K.R.B. 470 (2); Leite, P.F. 53 (2); Lima, C.T. 190,192 (1), 211(2), 223(1); Lima, R. 1686 (2); Lughadha, E.N. 6014 (1); Matos, R.M. 139, s.n. HUNEB 02181 (1); Mattos-Silva, D. 4783 (1); Melo, E. 2011 (2), 3157, 5290 (1); Moraes, M.V. 468 (2), 807 (1); Noblick, L.R. 1891 (1, 2), 2730 (2); Paciornik, E.F. 44 (1); Paixão, J.L. 1379 (1); Pinto, G.C.P. 201/84 (1); Plowman, T. 8942 (2); Queiroz, E.P. 1278 (1); L. Scardino 862 (2); Senra, L.C. 27 (2). 